International Journal of Pure and Applied Mathematics

Volume 102 No. 2 2015, 265-272

ISSN: 1311-8080 (printed version); ISSN: 1314-3395 (on-line version)

url: http://www.ijpam.eu

doi: http://dx.doi.org/10.12732/ijpam.v102i2.8

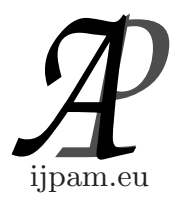

\title{
SYMMETRIC PROPERTIES FOR THE GENERALIZED \\ TWISTED $(h, q)$-EULER POLYNOMIALS \\ ASSOCIATED WITH $p$-ADIC INVARIANT \\ $q$-INTEGRAL ON $\mathbb{Z}_{p}$
}

\author{
C.S. Ryoo \\ Department of Mathematics \\ Hannam University \\ Daejeon, 306-791, KOREA
}

\begin{abstract}
In this paper, we study the symmetry for generalized twisted $(h, q)$-Euler numbers $E_{n, \chi, q, \zeta}^{(h)}$ and polynomials $E_{n, \chi, q, \zeta}^{(h)}(x)$. We obtain some interesting identities of the power sums and generalized twisted $(h, q)$-Euler polynomials $E_{n, \chi, q, \zeta}^{(h)}(x)$ using the symmetric properties for the $p$-adic invariant $q$-integral on $\mathbb{Z}_{p}$.
\end{abstract}

AMS Subject Classification: 11B68, 11S40, 11S80

Key Words: Euler numbers and polynomials, generalized twisted $(h, q)$-Euler numbers and polynomials, symmetric properties, power sums

\section{Introduction}

Throughout this paper we use the following notations. By $\mathbb{Z}_{p}$ we denote the ring of $p$-adic rational integers, $\mathbb{Q}$ denotes the field of rational numbers, $\mathbb{Q}_{p}$ denotes the field of $p$-adic rational numbers, $\mathbb{C}$ denotes the complex number field, and $\mathbb{C}_{p}$ denotes the completion of algebraic closure of $\mathbb{Q}_{p}$. Let $\nu_{p}$ be the 
normalized exponential valuation of $\mathbb{C}_{p}$ with $|p|_{p}=p^{-\nu_{p}(p)}=p^{-1}$. When one talks of $q$-extension, $q$ is considered in many ways such as an indeterminate, a complex number $q \in \mathbb{C}$, or $p$-adic number $q \in \mathbb{C}_{p}$. If $q \in \mathbb{C}$ one normally assume that $|q|<1$. If $q \in \mathbb{C}_{p}$, we normally assume that $|q-1|_{p}<p^{-\frac{1}{p-1}}$ so that $q^{x}=\exp (x \log q)$ for $|x|_{p} \leq 1$ (see [1-9]). Throughout this paper we use the notation:

$$
[x]_{q}=\frac{1-q^{x}}{1-q}, \quad[x]_{-q}=\frac{1-(-q)^{x}}{1+q} .
$$

Hence, $\lim _{q \rightarrow 1}[x]=x$ for any $x$ with $|x|_{p} \leq 1$ in the present $p$-adic case. Let $U D\left(\mathbb{Z}_{p}\right)$ be the space of uniformly differentiable function on $\mathbb{Z}_{p}$. For $g \in U D\left(\mathbb{Z}_{p}\right)$ the fermionic $p$-adic invariant $q$-integral on $\mathbb{Z}_{p}$ is defined by Kim as follows:

$$
I_{-q}(g)=\int_{\mathbb{Z}_{p}} g(x) d \mu_{-q}(x)=\lim _{N \rightarrow \infty} \frac{1}{\left[p^{N}\right]_{-q}} \sum_{x=0}^{p^{N}-1} g(x)(-q)^{x}, \text { see }[1,2] .
$$

If we take $g_{n}(x)=g(x+n)$ in $(1.1)$, then we see that

$$
q^{n} I_{q}\left(g_{n}\right)+(-1)^{n-1} I_{q}(g)=[2]_{q} \sum_{l=0}^{n-1}(-1)^{n-1-l} q^{l} g(l) .
$$

Let $T_{p}=\cup_{N \geq 1} C_{p^{N}}=\lim _{N \rightarrow \infty} C_{p^{N}}$, where $C_{p^{N}}=\left\{\zeta \mid \zeta^{p^{N}}=1\right\}$ is the cyclic group of order $p^{N}$. For $\zeta \in T_{p}$, we denote by $\phi_{\zeta}: \mathbb{Z}_{p} \rightarrow \mathbb{C}_{p}$ the locally constant function $x \longmapsto \zeta^{x}$ (see $\left.[7,8]\right)$.

Let $\chi$ be the primitive Dirichlet character with conductor $d \in \mathbb{N}$ with $d \equiv$ $1(\bmod 2)$ and $\zeta \in T_{p}$. We assume that $h \in \mathbb{Z}$ and $q \in \mathbb{C}_{p}$ with $|q-1|_{p}<1$. Let $g(y)=\chi(y) \phi_{\zeta}(y) q^{(h-1) y} e^{(y+x) t}$.

By (1.1), we derive

$$
\begin{aligned}
\int_{X} \chi(y) \phi_{\zeta}(y) q^{(h-1) y} e^{(y+x) t} d \mu_{-q}(y) & =\frac{[2]_{q} \sum_{a=0}^{d-1} \chi(a)(-1)^{a} \zeta^{a} q^{h a} e^{a t}}{\zeta^{d} q^{h d} e^{d t}+1} e^{x t} \\
& =\sum_{n=0}^{\infty} E_{n, \chi, q, \zeta}^{(h)}(x) \frac{t^{n}}{n !} .
\end{aligned}
$$

By using Taylor series of $e^{(y+x) t}$ in the above equation (1.3), we obtain

$$
\sum_{n=0}^{\infty}\left(\int_{X} \chi(y) \phi_{\zeta}(y) q^{(h-1) y}(y+x)^{n} d \mu_{-q}(y)\right) \frac{t^{n}}{n !}=\sum_{n=0}^{\infty} E_{n, \chi, q, \zeta}^{(h)}(x) \frac{t^{n}}{n !} .
$$


By comparing coefficients of $\frac{t^{n}}{n !}$ in the above equation, we have the Witt formula for the generalized twisted $(h, q)$ - Euler polynomials attached to $\chi$ as follows:

Theorem 1.1. For positive integers $n$ and $\zeta \in T_{p}$, we have

$$
E_{n, \chi, q, \zeta}^{(h)}(x)=\int_{X} \chi(y) \phi_{\zeta}(y)(y+x)^{n} d \mu_{-q}(y) .
$$

Observe that for $x=0$, the equation (1.4) reduces to (1.5).

Corollary 1.2. For positive integers $n$ and $\zeta \in T_{p}$, we have

$$
E_{n, \chi, q, \zeta}^{(h)}=\int_{X} \chi(y) y^{n} \phi_{\zeta}(y) d \mu_{-q}(y) .
$$

By (1.4) and (1.5), we obrian the following theorem.

Theorem 1.3. For positive integers $n$ and $\zeta \in T_{p}$, we have

$$
E_{n, \chi, q, \zeta}^{(h)}(x)=\sum_{l=0}^{n}\left(\begin{array}{l}
n \\
l
\end{array}\right) E_{l, \chi, q, \zeta}^{(h)} x^{n-l} .
$$

\section{Symmetry for the Generalized Twisted $(h, q)$-Euler Polynomials}

In this section, we assume that $q \in \mathbb{C}_{p}$ and $\zeta \in T_{p}$. We give some interesting identities of the power sums and generalized twisted $(h, q)$-Euler polynomials $E_{n, \chi, q, \zeta}^{(h)}(x)$ using the symmetric properties for the $p$-adic invariant $q$-integral on $\mathbb{Z}_{p}$. If $n$ is odd from (1.2), we obtain

$$
q^{n} I_{q}\left(g_{n}\right)+I_{q}(g)=[2]_{q} \sum_{l=0}^{n-1}(-1)^{l} q^{l} g(l) .
$$

Substituting $g(x)=\chi(x) \zeta^{x} q^{(h-1) x} e^{x t}$ into the above, we obtain

$$
\begin{aligned}
& \zeta^{n d} q^{h n d} \int_{X} \chi(x) \zeta^{x} q^{(h-1) x} e^{(x+n d) t} d \mu_{-q}(x)+\int_{X} \chi(x) \zeta^{x} q^{(h-1) x} e^{x t} d \mu_{-q}(x) \\
& =[2]_{q} \sum_{j=0}^{n d-1}(-1)^{j} \chi(j) \zeta^{j} q^{h j} e^{j t} .
\end{aligned}
$$


For $k \in \mathbb{N} \cup\{0\}$, let us define the $p$-adic functional $T_{k, \chi, q, \zeta}^{(h)}(n)$ as follows:

$$
T_{k, \chi, q, \zeta}^{(h)}(n)=\sum_{l=0}^{n}(-1)^{l} \chi(l) q^{h l} \zeta^{l} l^{k}
$$

After some elementary calculations, we have

$$
\begin{aligned}
& \zeta^{n d} q^{h n d} e^{n d t} \int_{X} \chi(x) \zeta^{x} q^{(h-1) x} e^{x t} d \mu_{-q}(x)+\int_{X} \chi(x) \zeta^{x} q^{(h-1) x} e^{x t} d \mu_{-q}(x) \\
& =\left(1+\zeta^{n d} q^{h n d} e^{n d t}\right) \frac{[2]_{q} \sum_{a=0}^{d-1} \chi(a)(-1)^{a} \zeta^{a} q^{h a} e^{a t}}{\zeta^{d} q^{h d} e^{d t}+1} .
\end{aligned}
$$

From the above, we get

$$
\begin{aligned}
q^{h n d} & \int_{X} \chi(x) \zeta^{x+n d} q^{(h-1) x} e^{(x+n d) t} d \mu_{-q}(x)+\int_{X} \chi(x) \zeta^{x} q^{(h-1) x} e^{x t} d \mu_{-q}(x) \\
= & \frac{[2]_{q} \int_{X} \chi(x) \zeta^{x} q^{(h-1) x} e^{x t} d \mu_{-q}(x)}{\int_{\mathbb{Z}_{p}} \zeta^{n d x} q^{(h n d-1) x} e^{n d t x} d \mu_{-q}(x)} .
\end{aligned}
$$

By substituting Taylor series of $e^{x t}$ into (2.2), we obtain

$$
\begin{aligned}
& \sum_{m=0}^{\infty}\left(\zeta^{n d} q^{h n d} \int_{X} \chi(x) \zeta^{x} q^{(h-1) x}(x+n d)^{m} d \mu_{-q}(x)\right. \\
& \left.\quad+\int_{X} \chi(x) \zeta^{x} q^{(h-1) x} x^{m} d \mu_{-q}(x)\right) \frac{t^{m}}{m !} \\
& =\sum_{m=0}^{\infty}\left([2]_{q} \sum_{j=0}^{n d-1}(-1)^{j} \chi(j) \zeta^{j} q^{h j} j^{m}\right) \frac{t^{m}}{m !} .
\end{aligned}
$$

By comparing coefficients $\frac{t^{m}}{m !}$ in the above equation, we obtain

$$
\begin{aligned}
& \zeta^{n d} q^{h n d} \sum_{k=0}^{m}\left(\begin{array}{c}
m \\
k
\end{array}\right)(n d)^{m-k} \int_{X} \chi(x) \zeta^{x} q^{(h-1) x} x^{k} d \mu_{-q}(x) \\
& \quad+\int_{X} \chi(x) \zeta^{x} q^{(h-1) x} x^{m} d \mu_{-q}(x)=[2]_{q} \sum_{j=0}^{n d-1}(-1)^{j} \chi(j) \zeta^{j} q^{h j} j^{m} .
\end{aligned}
$$


By using (2.3), we have

$$
\begin{aligned}
& \zeta^{n d} q^{h n d} \sum_{k=0}^{m}\left(\begin{array}{c}
m \\
k
\end{array}\right)(n d)^{m-k} \int_{X} \chi(x) \zeta^{x} q^{(h-1) x} x^{k} d \mu_{-q}(x) \\
& \quad+\int_{X} \chi(x) \zeta^{x} q^{(h-1) x} x^{m} d \mu_{-q}(x)=[2]_{q} T_{m, \chi, q, \zeta}^{(h)}(n d-1) .
\end{aligned}
$$

Hence, we arrive at the following theorem:

Theorem 2.1. Let $n$ be odd positive integer. Then we have

$$
\frac{\int_{X} \chi(x) \zeta^{x} q^{(h-1) x} e^{x t} d \mu_{-q}(x)}{\int_{\mathbb{Z}_{p}} \zeta^{n d x} q^{(h n d-1) x} e^{n d t x} d \mu_{-q}(x)}=\sum_{m=0}^{\infty}\left(T_{m, \chi, q, \zeta}^{(h)}(n d-1)\right) \frac{t^{m}}{m !} .
$$

Let $w_{1}$ and $w_{2}$ be odd positive integers. By Theorem 2.1 and after some elementary calculations, we have the following theorem.

Theorem 2.2. Let $w_{1}$ and $w_{2}$ be odd positive integers. Then we have

$$
\frac{\int_{X} \chi(x) \zeta^{w_{2} x} q^{\left(w_{2}-1\right) x} e^{w_{2} x t} d \mu_{-q}(x)}{\int_{\mathbb{Z}_{p}} \zeta^{w_{1} w_{2} d x} q^{\left(w_{1} w_{2} d-1\right) x} e^{w_{1} w_{2} t d x} d \mu_{-q}(x)}=\sum_{m=0}^{\infty}\left(T_{m, \chi, q, \zeta^{w_{2}}}^{\left(w_{2}\right)}\left(w_{1} d-1\right) w_{2}^{m}\right) \frac{t^{m}}{m !} .
$$

Then we set

$S\left(w_{1}, w_{2}\right)=$

$\frac{\int_{X} \int_{X} \chi\left(x_{1}\right) \chi\left(x_{2}\right) \zeta^{\left(w_{1} x_{1}+w_{2} x_{2}\right)} q^{\left(w_{1}-1\right) x_{1}} q^{\left(w_{2}-1\right) x_{2}} e^{\left(w_{1} x_{1}+w_{2} x_{2}+w_{1} w_{2} x\right) t} d \mu_{-q}\left(x_{1}\right) d \mu_{-q}\left(x_{2}\right)}{\int_{\mathbb{Z}_{\mathrm{p}}} \zeta^{w_{1} w_{2} d x} q^{\left(w_{1} w_{2} d-1\right) x} e^{w_{1} w_{2} d x t} d \mu_{-q}(x)}$.

By Theorem 2.2 and $S\left(w_{1}, w_{2}\right)$, after elementary calculations, we have

$$
\begin{aligned}
S\left(w_{1}, w_{2}\right)= & \left(\int_{X} \chi\left(x_{1}\right) \zeta^{w_{1} x_{1}} q^{\left(w_{1}-1\right) x_{1}} e^{\left(w_{1} x_{1}+w_{1} w_{2} x\right) t} d \mu_{-q}\left(x_{1}\right)\right) \\
& \times\left(\frac{\int_{X} \chi\left(x_{2}\right) \zeta^{w_{2} x_{2}} q^{\left(w_{2}-1\right) x_{2}} e^{x_{2} w_{2} t} d \mu_{-q}\left(x_{2}\right)}{\int_{\mathbb{Z}_{p}}^{\zeta^{w_{1} w_{2} d x}} q^{\left(w_{1} w_{2} d-1\right) x} e^{w_{1} w_{2} d x t} d \mu_{-q}(x)}\right) \\
= & \left(\sum_{m=0}^{\infty} E_{m, \chi, q, \zeta^{w_{1}}}^{\left(w_{1}\right)}\left(w_{2} x\right) w_{1}^{m} \frac{t^{m}}{m !}\right)\left(\sum_{m=0}^{\infty} T_{m, \chi, q, \zeta^{w_{2}}}^{\left(w_{2}\right)}\left(w_{1} d-1\right) w_{2}^{m} \frac{t^{m}}{m !}\right) .
\end{aligned}
$$


By using Cauchy product in the above, we have

$$
S\left(w_{1}, w_{2}\right)=\sum_{m=0}^{\infty}\left(\sum_{j=0}^{m}\left(\begin{array}{c}
m \\
j
\end{array}\right) E_{j, \chi, q, \zeta^{w_{1}}}^{\left(w_{1}\right)}\left(w_{2} x\right) w_{1}^{j} T_{m-j, \chi, q, \zeta^{w}}^{\left(w_{2}\right)}\left(w_{1} d-1\right) w_{2}^{m-j}\right) \frac{t^{m}}{m !} .
$$

From the symmetry of $S\left(w_{1}, w_{2}\right)$ in $w_{1}$ and $w_{2}$, we also see that

$$
\begin{aligned}
S\left(w_{1}, w_{2}\right)= & \left(\int_{X} \chi\left(x_{2}\right) \zeta^{w_{2} x_{2}} q^{\left(w_{2}-1\right) x_{2}} e^{\left(w_{2} x_{2}+w_{1} w_{2} x\right) t} d \mu_{-q}\left(x_{2}\right)\right) \\
\times & \times\left(\frac{\int_{X} \chi\left(x_{1}\right) \zeta^{w_{1} x_{1}} q^{\left(w_{1}-1\right) x_{1}} e^{x_{1} w_{1} t} d \mu_{-q}\left(x_{1}\right)}{\int_{\mathbb{Z}_{p}} \zeta^{w_{1} w_{2} d x} q^{\left(w_{1} w_{2} d-1\right) x} e^{w_{1} w_{2} d x t} d \mu_{-q}(x)}\right) \\
= & \left(\sum_{m=0}^{\infty} E_{m, \chi, q, \zeta^{w_{2}}}^{\left(w_{2}\right)}\left(w_{1} x\right) w_{2}^{m} \frac{t^{m}}{m !}\right)\left(\sum_{m=0}^{\infty} T_{m, \chi, q, \zeta^{w_{1}}}^{\left(w_{1}\right)}\left(w_{2} d-1\right) w_{1}^{m} \frac{t^{m}}{m !}\right) .
\end{aligned}
$$

Thus we have

$$
S\left(w_{1}, w_{2}\right)=\sum_{m=0}^{\infty}\left(\sum_{j=0}^{m}\left(\begin{array}{c}
m \\
j
\end{array}\right) E_{j, \chi, q, \zeta^{w_{2}}}^{\left(w_{2}\right)}\left(w_{1} x\right) w_{2}^{j} T_{m-j, \chi, q, \zeta^{w_{1}}}^{\left(w_{1}\right)}\left(w_{2} d-1\right) w_{1}^{m-j}\right) \frac{t^{m}}{m !} .
$$

Thus we arrive at the following theorem:

Theorem 2.3. Let $w_{1}$ and $w_{2}$ be odd positive integers. Then we obtain

$$
\begin{aligned}
& \sum_{j=0}^{m}\left(\begin{array}{c}
m \\
j
\end{array}\right) w_{1}^{m-j} w_{2}^{j} E_{j, \chi, q, \zeta^{w_{2}}}^{\left(w_{2}\right)}\left(w_{1} x\right) T_{m-j, \chi, q, \zeta^{w_{1}}}^{\left(w_{1}\right)}\left(w_{2} d-1\right) \\
& =\sum_{j=0}^{m}\left(\begin{array}{c}
m \\
j
\end{array}\right) w_{1}^{j} w_{2}^{m-j} E_{j, \chi, q, \zeta^{w_{1}}}^{\left(w_{1}\right)}\left(w_{2} x\right) T_{m-j, \chi, q, \zeta^{w_{2}}}^{\left(w_{2}\right)}\left(w_{1} d-1\right),
\end{aligned}
$$

where $E_{k, \chi, q, \zeta}^{\left(w_{1}\right)}(x)$ and $T_{m, \chi, q, \zeta}^{\left(w_{1}\right)}(k)$ denote generalized twisted $(h, q)$-Euler polynomials and $p$-adic functional, respectively.

By Theorem 2.3, we have the following corollary.

Corollary 2.4. Let $w_{1}$ and $w_{2}$ be odd positive integers. Then we obtain

$$
\begin{aligned}
& \sum_{j=0}^{m} \sum_{k=0}^{j}\left(\begin{array}{c}
m \\
j
\end{array}\right)\left(\begin{array}{l}
j \\
k
\end{array}\right) w_{1}^{m-k} w_{2}^{j} x^{j-k} E_{k, \chi, q, \zeta^{w}}^{\left(w_{2}\right)} T_{m-j, \chi, q, \zeta^{w_{1}}}^{\left(w_{1}\right)}\left(w_{2} d-1\right) \\
& =\sum_{j=0}^{m} \sum_{k=0}^{j}\left(\begin{array}{c}
m \\
j
\end{array}\right)\left(\begin{array}{l}
j \\
k
\end{array}\right) w_{1}^{j} w_{2}^{m-k} x^{j-k} E_{k, \chi, q, \zeta^{w}}^{\left(w_{1}\right)} T_{m-j, \chi, q, \zeta^{w_{2}}}^{\left(w_{2}\right)}\left(w_{1} d-1\right) .
\end{aligned}
$$


Now we will derive another interesting identities for generalized twisted $(h, q)$-Euler polynomials using the symmetric property of $S\left(w_{1}, w_{2}\right)$.

$$
\begin{aligned}
& S\left(w_{1}, w_{2}\right) \\
& =\sum_{j=0}^{w_{1} d-1}(-1)^{j} \chi(j) q^{w_{2} j} \int_{X} \chi\left(x_{1}\right) \zeta^{w_{1} x_{1}} q^{\left(w_{1}-1\right) x_{1}} e^{\left(x_{1}+w_{2} x+j \frac{w_{2}}{w_{1}}\right)\left(w_{1} t\right)} d \mu_{-q}\left(x_{1}\right) \\
& =\sum_{n=0}^{\infty}\left(\sum_{j=0}^{w_{1} d-1}(-1)^{j} \chi(j) \zeta^{w_{2} j} q^{w_{2} j} E_{n, \chi, q, \zeta^{w}}^{\left(w_{1}\right)}\left(w_{2} x+j \frac{w_{2}}{w_{1}}\right) w_{1}^{n}\right) \frac{t^{n}}{n !} .
\end{aligned}
$$

By using the symmetry property in the above equation, we also have

$$
\begin{aligned}
& S\left(w_{1}, w_{2}\right) \\
& =\sum_{j=0}^{w_{2} d-1}(-1)^{j} \chi(j) \zeta^{w_{1} j} q^{w_{1} j} \int_{X} \chi\left(x_{2}\right) \zeta^{w_{2} x_{2}} q^{\left(w_{2}-1\right) x_{2}} e^{\left(x_{2}+w_{1} x+j \frac{w_{1}}{w_{2}}\right)\left(w_{2} t\right)} d \mu_{-q}\left(x_{2}\right) \\
& =\sum_{n=0}^{\infty}\left(\sum_{j=0}^{w_{2} d-1}(-1)^{j} \chi(j) \zeta^{w_{1} j} q^{w_{1} j} E_{n, \chi, q, \zeta^{w}}^{\left(w_{2}\right)}\left(w_{1} x+j \frac{w_{1}}{w_{2}}\right) w_{2}^{n}\right) \frac{t^{n}}{n !} .
\end{aligned}
$$

Thus we obtain the following theorem.

Theorem 2.5. Let $w_{1}$ and $w_{2}$ be odd positive integers and $\zeta \in T_{p}$. Then we obtain

$$
\begin{aligned}
& \sum_{j=0}^{w_{1} d-1}(-1)^{j} \chi(j) \zeta^{w_{2} j} q^{w_{2} j} E_{n, \chi, q, \zeta^{w_{1}}}^{\left(w_{1}\right)}\left(w_{2} x+j \frac{w_{2}}{w_{1}}\right) w_{1}^{n} \\
= & \sum_{j=0}^{w_{2} d-1}(-1)^{j} \chi(j) \zeta^{w_{1} j} q^{w_{1} j} E_{n, \chi, q, \zeta^{w_{2}}}^{\left(w_{2}\right)}\left(w_{1} x+j \frac{w_{1}}{w_{2}}\right) w_{2}^{n} .
\end{aligned}
$$

If we take $x=0$ in Theorem 2.5, we also derive the interesting identity for generalized twisted $(h, q)$-Euler numbers as follows: 
Corollary 2.6. Let $w_{1}$ and $w_{2}$ be odd positive integers. Then we obtain

$$
\begin{aligned}
& \sum_{j=0}^{w_{1} d-1}(-1)^{j} \chi(j) \zeta^{w_{2} j} q^{w_{2} j} E_{n, \chi, q, \zeta^{w_{1}}}^{\left(w_{1}\right)}\left(\frac{j w_{2}}{w_{1}}\right) w_{1}^{n} \\
= & \sum_{j=0}^{w_{2} d-1}(-1)^{j} \chi(j) \zeta^{w_{1} j} q^{w_{1} j} E_{n, \chi, q, \zeta^{w_{2}}}^{\left(w_{2}\right)}\left(\frac{j w_{1}}{w_{2}}\right) w_{2}^{n} .
\end{aligned}
$$

\section{References}

[1] T. Kim, q-Volkenborn integration, Russ. J. Math. Phys., 9(2002), 288-299.

[2] T. Kim, $q$-Euler numbers and polynomials associated with $p$-adic $q$ integrals, J. Nonlinear Math. Phys., 14(2007), 15-27.

[3] T. Kim, J. Choi, Y-.H. Kim, C. S. Ryoo, A Note on the weighted $p$-adic $q$-Euler measure on $\mathbb{Z}_{p}$, Advan. Stud. Contemp. Math., 21(2011), 35-40.

[4] C. S. Ryoo, T. Kim, L.-C. Jang, Some relationships between the analogs of Euler numbers and polynomials, J. Inequal. Appl., 2007(2007), Art ID 86052, 22pp.

[5] C. S. Ryoo, On the generalized Barnes type multiple $q$-Euler polynomials twisted by ramified roots of unity, Proc. Jangjeon Math. Soc., 13(2010), $255-263$.

[6] C. S. Ryoo, A note on the weighted $q$-Euler numbers and polynomials, Advan. Stud. Contemp. Math., 21(2011), 47-54.

[7] C. S. Ryoo, On the Generalized Twisted $q$-Euler Numbers and Polynomials with Weak Weight $\alpha$, Adv. Studies Theor. Phys., 7(5)(2013), 245 - 251

[8] C. S. Ryoo, A Note on the Twisted $q$-Euler Numbers and Polynomials with Weak Weight $\alpha$, Adv. Studies Theor. Phys., 6(2012), 1109 - 1116.

[9] C. S. Ryoo, An Identity of the $(h, q)$-Euler Polynomials Associated with the $p$-Adic $q$-Integrals on $\mathbb{Z}_{p}$, Int. Journal of Math. Analysis, 7(2013), 315 -321 . 